\title{
Michael Fischer
}

\section{Erfahrungen aus der Ausbildungspraxis der Badischen Landesbibliothek Experiences in the training practice at the Baden State Library}

http://doi.org/10.1515/bd-2018-0102

Zusammenfassung: Ausgehend von der aktuellen Situation der bibliothekarischen Ausbildung in den verschiedenen Qualifikationsniveaus skizziert der Beitrag die im letzten Jahr an der Badischen Landesbibliothek erfolgten Änderungen der Ausbildungsinhalte und -organisation.

Schlüsselwörter: Praktische Ausbildung, Praxisrelevanz, Arbeitsmarkt

Abstract: Taking the current situation in the training of future librarians on various levels of qualification as a starting point, the article outlines the changes in the content and organisation of the training programme during the past year at Baden State Library.

Keywords: practical training, practical relevance, labour market

\section{Einleitung}

Wohin sich die bibliothekarische Zukunft angesichts der durch die Digitalisierung hervorgerufenen Herausforderungen entwickeln wird, ist an vielen Stellen noch unklar. Zweifelsohne jedoch befindet sich der gesamte Berufsstand im Umbruch und damit auch und in besonderem Maße die Ausbildung. ${ }^{1}$ Dieser Beitrag reflektiert die Erfahrungen der Badischen Landesbibliothek, die lokale Ausbildungspraxis an die durch diesen Umbruch ausgelösten Erfordernisse anzupassen insbesondere wie dabei die in Folge der Digitalisierung in den Bibliotheken

1 Vgl. Söllner, Konstanze: Bibliotheken ohne Bibliothekar/innen? Qualifikationen für die wissenschaftliche Bibliothek. In: Bibliotheksdienst 51 (2017), Heft 10-11, S. 852-863, hier: S. 852.

Michael Fischer: fischer@blb-karlsruhe.de 
entstandenen „neuen Tätigkeiten“2 unter der Maßgabe der Praxis- bzw. Arbeitsmarktrelevanz in die Ausbildung integriert wurden. Dabei werden eingangs nochmals - gegliedert nach den verschiedenen Qualifikationsniveaus - die aktuellen Probleme in der bibliothekarischen Ausbildung skizziert und anschließend die Ergänzungen der Ausbildungsinhalte sowie laufbahnspezifische Anpassungen und Änderungen in der Ausbildungsorganisation an der BLB geschildert.

\section{Aktuelle Situation der Ausbildung zur/m Fach- angestellten für Medien- und Informations- dienste}

Seit einigen Jahren wird es immer schwieriger, sowohl freiwerdende Stellen als auch Ausbildungsplätze für Fachangestellte für Medien- und Informationsdienste adäquat zu besetzen. ${ }^{3}$ Dabei schätzen in einer Umfrage des dbv-Landesverbandes Baden-Württemberg knapp zwei Drittel der antwortenden wissenschaftlichen Bibliotheken das „Angebot bei Bewerbungen“ für die mittlere Qualifikationsebene als nur „ausreichend“ bzw. „mangelhaft“ ein. ${ }^{4}$ Diese Situation wird sich

2 Als „neue Tätigkeiten“ sollen hier - unabhängig vom Qualifikationsniveau - alle durch die Herausforderung der Digitalisierung vor allem im letzten Jahrzehnt entstandenen beruflichen Tätigkeiten in Bibliotheken verstanden werden. Vgl. wie Anm. 1. Vgl. BIB Hamburg (Hrsg.): Auswertung der Befragung zum Thema „neue/andere Tätigkeiten für FAMIs“. URL: http://www. bib-info.de/fileadmin/media/Dokumente/Landesgruppen/Hamburg/hamburg/Protokolle/Auswertung_FAMI_Taetigkeiten.pdf [Zugriff: 08. 08. 2018]. Vgl. Holste-Flinspach, Karin: Der FaMIBeruf. Tätigkeitsfelder und Berufswege. In: Bibliotheksdienst 51 (2017), Heft 10-11, S. 943-950. Vgl. Bonte, Achim: Der Wissenschaftliche Dienst in der Digitalen Bibliothek. Was kommt nach dem Fachreferentensystem? URL: https://www.slideshare.net/Achim_Bonte/der-wissenschaftliche-dienst-in-der-digitalen-bibliothek-was-kommt-nach-dem-fachreferentensystem-39650127 [Zugriff: 08. 08. 2018].

3 Vgl. dbv Baden-Württemberg (Hrsg.): Umfrage des dbv Landesverbandes Baden-Württemberg. Fachkräfte in baden-württembergischen Bibliotheken aller Sparten - Situation bei Stellenbesetzungen und Ausbildungsverträgen, S. 1. URL: https://www.bibliotheksverband.de/fileadmin/ user_upload/Landesverbaende/Baden-Wuerttemberg/2017-12_Fragebogen.pdf [Zugriff: 08. 08. 2018]. Vgl. zur Umfrage des dbv Landesverbandes Baden-Württemberg: Engpässe bei Stellenbesetzungen und Nachwuchsgewinnung. In: BuB 70 (2018), Heft 8-9, S. $474 \mathrm{f}$.

4 Vgl. dbv Baden-Württemberg (Hrsg.): Fachkräfte in baden-württembergischen Bibliotheken aller Sparten. Situation bei Stellenbesetzungen und Ausbildungsverträgen. Umfrage des dbv Landesverbands Sommer 2017, S. 9. URL: https://www.bibliotheksverband.de/fileadmin/ user_upload/Landesverbaende/Baden-Wuerttemberg/2017-12_Auswertung_Umfrage_final.pdf [Zugriff: 08. 08. 2018]. 
in den kommenden Jahren durch die demographische Entwicklung tendenziell noch verschärfen. ${ }^{5}$

Dies mag damit zusammenhängen, dass die gegenwärtig praktizierte FaMIAusbildung nicht in allen Bereichen die notwendige Praxisrelevanz aufweist, d.h. die Ausbildung nicht konsequent an den Erfordernissen des Arbeitsmarktes ausgerichtet ist, was durch eine Umfrage der BIB-Landesgruppe Hamburg vom Herbst 2015 unterstrichen wird. ${ }^{6}$ Diese untersuchte, in welchen „neuen Tätigkeitsbereichen“ FaMIs in der betrieblichen Praxis mittlerweile eingesetzt werden: $28 \%$ der befragten wissenschaftlichen Bibliotheken setzen FaMIs im Schulungsbereich ein, 26\% in der Erwerbung, $25 \%$ in der Erschließung und immerhin 13\% im Bereich der Digitalisierung, ohne dass jedoch diese Bereiche umfangreich in der Ausbildung vermittelt werden.

Obwohl die Anzahl der begonnenen FaMI-Ausbildungen mit jährlich bundesweit ca. 600 in etwa konstant ist, ist die Anzahl der Bewerber/innen in den vergangenen Jahren zurückgegangen, vor allem in den urbanen Einzugsbereichen der meisten wissenschaftlichen Bibliotheken. ${ }^{7}$ Wenngleich dies kein spezifisches Problem der FaMI-Ausbildung ist, sondern in vielen Bereichen der beruflichen Ausbildung auftritt, wiegt es aufgrund des relativ geringen Bekanntheitsgrades des Berufes ungleich schwerer: Im Ausbildungsjahr 2015/16 konnten in BadenWürttemberg bereits 14 Ausbildungsplätze nicht besetzt werden. ${ }^{8}$

Noch sehr oft begründen die Bewerber/innen die Aufnahme einer FaMI-Ausbildung mit dem Interesse an Büchern ${ }^{9}$ bzw. ziehen die gegenwärtigen Ausschreibungen und das gegenwärtig in der Öffentlichkeit bekannte Berufsbild vor allem „Buchmenschen“ an. ${ }^{10}$ Obschon ein hohes Interesse am Medium Buch natürlich ein guter Grund für die Arbeit in einer Bibliothek sein kann, müssen Bibliotheken, wenn der Maßstab der Arbeitsmarktrelevanz angelegt wird, künftig zwin-

5 Vgl. Holste-Flinspach, Karin: Der FaMI-Beruf. Tätigkeitsfelder und Berufswege. In: Bibliotheksdienst 51 (2017), Heft 10-11, S. 943-950, hier: S. 944.

6 BIB Hamburg (Hrsg.): Auswertung der Befragung zum Thema „neue/andere Tätigkeiten für FAMIs“, S. 5. URL: http://www.bib-info.de/fileadmin/media/Dokumente/Landesgruppen/Hamburg/hamburg/Protokolle/Auswertung_FAMI_Taetigkeiten.pdf [Zugriff: 08. 08. 2018]. Allerdings setzen gleichzeitig nur 5\% der befragten Bibliotheken FaMIs auch im Bereich der Lizenzverwaltung von E-Medien bzw. $2 \%$ bei elektronischen Angeboten ein.

7 Vgl. wie Anm. 5, S. 944.

8 Vgl. wie Anm. 4, S. 12.

9 Vgl. wie Anm. 5, S. 944. Vgl. Schleihagen, Barbara: Wie gewinnen wir neues Personal für veränderte Bibliotheken? In: BuB 70 (2018), Heft 8-9, S. 470-473, hier: S. 470.

10 Vgl. Positionspapier „Berufsbild und Entwicklung“. In: Bibliotheksdienst 51 (2017), Heft 10-11, S. 876-877, hier: S. 877. 
gend in hohem Maße IT-affine sowie kommunikationsorientierte Bewerber/innen ansprechen. ${ }^{11}$

Die allermeisten Bewerber/innen für FaMI-Ausbildungen an wissenschaftlichen Bibliotheken verfügen mittlerweile über die allgemeine Hochschulreife, oftmals sind zudem Studienabbrecher/innen bzw. sogar -absolvent/inn/en stark vertreten. ${ }^{12}$ Auch hier spiegelt die Situation der FaMI-Ausbildung lediglich einen allgemeinen Trend wider, den die überdurchschnittliche Bereitschaft wissenschaftlicher Bibliotheken, Ausbildungsstellen mit Bewerber/inne/n mit höherem Bildungsabschluss zu besetzen, noch verstärkt. Dies ist deswegen problematisch, weil die Auszubildenden dann im obligatorischen Berufsschulunterricht sowie auch im Ausbildungs- bzw. späteren Berufsalltag unterfordert werden oder im Anschluss an die dreijährige Ausbildung ein LIS-Studium beginnen, ${ }^{13}$ das ja bereits vor der Ausbildung hätte aufgenommen werden können. Dadurch werden die Ausbildungskapazitäten aller Beteiligten über das notwendige Maß hinaus strapaziert. Nur eine Minderheit der Bewerber/innen verfügt hingegen über den für eine Berufsausbildung ausreichenden Haupt- bzw. Realschulabschluss.

Die Zusammenarbeit zwischen Ausbildungsstätten und Berufsschulen ist dabei nicht immer ausreichend intensiv; inhaltlich kommen insbesondere die dort zu vermittelnden Regelwerkskenntnisse für die bibliothekarische Erschließung zu kurz.

\section{Aktuelle Situation bei den LIS-Studierenden}

Die Qualifikation von Absolvent/inn/en bibliothekarischer B.A.-Studiengänge wird von den einstellenden Bibliotheken ähnlich negativ beurteilt wie bei der mittleren Qualifikationsebene. ${ }^{14}$ Schon während der Umstellung von der verwaltungsinternen Ausbildung auf B.A.-Studiengänge wurde kritisiert, dass die Absolvent/inn/en nicht ohne weitere, umfangreiche Einarbeitung in den bibliothekarischen Kernbereichen einsetzbar seien sowie dass durch den großen Anteil von Managementinhalten falsche Erwartungen in Bezug auf die Berufspraxis geschürt würden, die realiter weniger aus Führungsaufgaben denn aus Sachbearbeitung

11 Vgl. Schleihagen, Barbara: Wie gewinnen wir neues Personal für veränderte Bibliotheken?. In: BuB 70 (2018), Heft 8-9, S. 470-473, hier: S. 470.

12 Vgl. Holste-Flinspach, Karin: Der Wettbewerb um gute Auszubildende ist in vollem Gang. In: BuB 70 (2018), Heft 8-9, S. 480-483, hier: S. 482.

13 Vgl. wie Anm. 1, S. 860.

1410 von 19 befragten Bibliotheken bezeichneten die Bewerber/innen-Lage beim gehobenen Dienst als „ausreichend“ oder mangelhaft, vgl. wie Anm. 4, S. 9. 
bestehe. ${ }^{15}$ In den meisten Bibliotheken befinden sich die einst dominierenden Tätigkeitsbereiche des gehobenen Dienstes - Erwerbung und Erschließung von Printmedien - auf dem Rückzug bzw. werden von FaMIs übernommen, ${ }^{16}$ so dass die Absolvent/inn/en der B.A.-Studiengänge ihren Einsatz künftig vorwiegend im Schulungsbereich, in leitenden Funktionen der Altbestandsdigitalisierung, im Lizenz-, Metadaten- und Erschließungsmanagement, bei systembibliothekarischen Aufgaben sowie bei der Unterstützung von forschungsnahen Diensten (wie Open Access, Publikations- oder Forschungsdatenmanagement oder Bibliometrie) finden werden. ${ }^{17}$

Dabei haben von allen theorievermittelnden Ausbildungsangeboten die Mehrzahl der B.A.-Studiengänge ihre Inhalte am konsequentesten und stringentesten den arbeitsmarktrelevanten „neuen Tätigkeitsbereichen“ angepasst: Die Umwandlung der Fakultät „Information und Kommunikation“ an der Hochschule der Medien in Stuttgart zur „transformativen Fakultät“ impliziert beispielweise auch die grundlegende und auf den Erwerb von Schlüsselkompetenzen ausgerichtete Umstellung des dort angebotenen bibliothekarischen B.A.-Studiengangs. ${ }^{18}$ Innerhalb des bibliothekarisch-fachlichen Curriculums des B.A. „Bibliotheks- und Informationsmanagement“ können sich die Studierenden ab WS 2018/19 in zwei Bereichen profilieren, zum einen im Profil „Bibliotheks-, Kulturund Bildungsmanagement“, zum anderen im Profil „Daten- und Informationsmanagement“, wobei die gemeinsam zu absolvierenden Inhalte mit einem Anteil von $40 \%$ relativ gering sind. Alle Fachstudierenden werden in den ersten beiden Semestern in den Modulen „IT-Grundlagen und Einführung in das Programmieren“ sowie „Web-Technologien“ unterrichtet, im dritten und vierten Semester in den Modulen „Open Society“ und „Cultural Heritage, Digitalisierung“. Im Profil „Daten- und Informationsmanagement“ werden in den folgenden Semestern weitere Themen aus den „neuen Tätigkeitsbereichen“ mit den Modulen „Daten und Datenanalyse“, „Web-Programmierung“ und „Metadatenmanagement“ intensiv vertieft. ${ }^{19}$ Auch andere akademische Ausbildungseinrichtungen haben

15 Vgl. Koelges, Barbara: Der Praxisbezug in den Bachelorstudiengängen. In BuB 62 (2010), Heft 1, S. 23-24, hier: S. 24. Vgl. wie Anm. 1, S. 861 f.

16 Vgl. Holste-Flinspach, Karin: FaMI-Einsatz der Zukunft. In: BuB 65 (2013), Heft 7-8, S. 525- 526.

17 Vgl. wie Anm. 1, S. 862.

18 Vgl. Vonhof, Cornelia: Bachelor und Berufspraxis - das Studienmodell der Hochschule der Medien Stuttgart. In: Bibliotheksdienst 51 (2017), Heft 10-11, S. 923-934, hier: S. 927 f.

19 Vgl. Vonhof, Cornelia: Bibliotheken und Hochschulen in einem Boot?! Trends in Bibliotheken und die Konsequenzen für die Lehre. Konferenzveröffentlichung 107. Deutscher Bibliothekartag in Berlin 2018. URN: urn:nbn:de:0290-opus4-157486 [Zugriff: 08. 08. 2018]. Vgl. auch wie Anm. 18, S. 930-932. 
ihre Curricula in diesem Sinne erweitert, so beispielsweise der verwaltungsinterne B.A.-Studiengang an der Hochschule für den öffentlichen Dienst in Bayern $^{20}$ oder der B.A.-Studiengang „Bibliothekswissenschaft“ an der FH Potsdam. ${ }^{21}$ Es bleibt zu hoffen, dass solchermaßen zukunftsorientiert ausgestattete Studiengänge auch die dafür passenden, d.h. im weitesten Sinne IT-affinen Studierenden werden anziehen können. Leider geben die bisherigen Erfahrungen diesbezüglich wenig Anlass zu Optimismus. ${ }^{22}$

\section{Aktuelle Situation bei der Ausbildung zur/m wissenschaftlichen Bibliothekar/in}

Nach wie vor sind die Bewerbungszahlen für eine postgraduale Ausbildung (Referendariat, Volontariat) zur/m wissenschaftlichen Bibliothekar/in hoch, wenngleich nach den ausgeschriebenen Fachrichtungen stark schwankend. Die grundsätzlich geeigneten Bewerber/inne/n sind, vor allem im Bereich der Geistes- und Sozialwissenschaften, Personen mit ausgewiesener fachlicher Expertise. Weit seltener besitzen diese auch die so sehr benötigten und von den einstellenden Bibliotheken gewünschten „Spezialistenkenntnisse“23 in den „neuen Tätigkeitsbereichen“. Diese Qualifikationen sind in den Stellenausschreibungen und Bewerber/innen-Informationen oft vage formuliert, so dass das

20 Wenngleich der Anteil der Bereiche, die den „neuen Tätigkeiten“ zuzuordnen sind in dem explizit auf wissenschaftliche Bibliotheken limitierten B.A.-Studiengang mit ca. 8-10\% relativ gering ausfällt. Vgl. Studiengang Bibliotheks- und Informationsmanagement. Modulhandbuch für den verwaltungsinternen Bachelor-Studiengang Bibliotheks- und Informationsmanagement am Fachbereich Archiv- und Bibliothekswesen der FHVR (3. Qualifizierungsstufe), S. $7 \mathrm{f}$, URL: http://www.fhvr-aub.bayern.de/fileadmin/user_upload/archiv_bib/studium/Modulhandbuch_2015-09-11.pdf [Zugriff: 08. 08. 2018].

21 Hier entfallen im Pflichtbereich des B.A.-Studiengangs ca. $13 \%$ und ggf. weitere $6 \%$ aus dem Wahlpflichtbereich auf Bereiche, die den ,neuen Tätigkeiten“ zuzuordnen sind. Vgl. Fachbereich Informationswissenschaften. Modulhandbuch Bibliothekswissenschaft (Bachelor of Arts), URL: https://www.fh-potsdam.de/fileadmin/user_dateien/2_studieren-FB_Infowiss/studium/modulhandbuecher/FB5_Modulhandbuch_Bibliothekswissenschaft-BA.pdf [Zugriff: 08. 08. 2018].

22 Vgl. wie Anm. 1, S. 862f. Vgl. auch die Erfahrungen des - allerdings sehr informatiklastigen - B.A.-Studiengangs „Bibliotheksinformatik“ an der HTWK Leipzig: Frank, Michael: Studienrichtung Bibliotheksinformatik in Leipzig - Intentionen und erste Erfahrungen. URL: http:// www.imn.htwk-leipzig.de/ mfrank/Wien_Sept_2015_Bibliotheksinformatik_lang.pdf [Zugriff: 08. 08. 2018]. Vgl. auch Susanne Krüger: Blick zurück in die Zukunft. In: BuB 68 (2016), Heft 6, S. 352-355.

23 Wie Anm. 1, S. 856. 
absolvierte Studium in der ausgeschriebenen Fachrichtung und ggf. eine Reihe von Schlüsselkompetenzen nach wie vor die einzig belastbaren Zugangskriterien sind. ${ }^{24}$ In der Praxis sind für die ausbildenden Bibliotheken jedoch die zusätzlich zum Fachstudium erworbenen Qualifikationen bzw. besonderen Erfahrungen der Bewerber/innen interessant. Dies bezieht sich nicht nur auf die allenthalben geforderten „IT-Kenntnisse“, sondern auch auf andere berufliche Vorerfahrungen, z.B. wissenschaftliche Tätigkeiten im Bereich der Digital Humanities, journalistische Fertigkeiten, Erfahrungen im Projekt- und Veranstaltungsmanagement sowie der Presse- und Öffentlichkeitsarbeit oder Lehrerfahrungen. Zudem ist es für die einstellenden Institutionen schwierig, die für eine spätere Tätigkeit im wissenschaftlichen Bibliotheksdienst zentralen Sozial- und Führungskompetenzen in einem Vorstellungsgespräch klassischen Zuschnitts vor Ort transparent abzuprüfen. Einen interessanten Weg, die Auswahl der Bewerber/innen für den wissenschaftlichen Bibliotheksdienst auf eine neue Grundlage zu stellen, ist seit 2010 die bayerische Ausbildung mit der Einführung von Assessment Centern gegangen. ${ }^{25}$ Diese dienen der Überprüfung der Schlüsselkompetenzen der Bewerber/innen, wobei davon ausgegangen wird, dass diese beim Eintritt in die postgraduale Ausbildung vorhanden sein müssen und nicht mehr durch Ausbildung vermittelt werden können. Es wäre zu prüfen, inwieweit die Auswahlmethode des Assessment Centers auch in anderen Bundesländern sinnvoll zum Einsatz kommen kann.

Allgemein herrscht in der Berufsbilddebatte „Ratlosigkeit“, ${ }^{26}$ was das Berufsbild (und damit auch die Ausbildung) des wissenschaftlichen Bibliotheksdienstes künftig ausmachen wird. ${ }^{27}$ Das 2014 veröffentliche VDB-Positionspapier zum Berufsbild des wissenschaftlichen Bibliothekars reihte „pragmatisch“ sowohl die

24 Vgl. Bibliotheksakademie Bayern (Hrsg.): Die Ausbildung für die vierte Qualifikationsebene mit Schwerpunkt Bibliothekswesen (ehem. höherer Bibliotheksdienst). URL: https://www.bsbmuenchen.de/fileadmin/pdf/bibliotheksakademie/q4_infoblatt_201605.pdf [Zugriff: 08. 08. 2018]. Vgl. Württembergische Landesbibliothek (Hrsg.): Merkblatt über die Ausbildung für den höheren Dienst an wissenschaftlichen Bibliotheken. URL: http://www.wlb-stuttgart.de/die-wlb/ ausbildung/merkblatt-hoeherer-dienst/ [Zugriff: 08. 08. 2018].

Vgl. Sächsische Landes- und Universitätsbibliothek (Hrsg.): Volontär/in. Bibliotheksvolontariat in Sachsen. URL: https://www.slub-dresden.de/ueber-uns/organisation/karriere-und-ausbildung/ausbildung/volontaerin/ [Zugriff: 08. 08. 2018]. Hier wird zudem auf die gewünschte „hohe IT-Kompetenz“ hingewiesen.

25 Vgl. Baumeister, Harald; Werr, Naoka: Das Assessment Center als Suche nach dem oder der Richtigen. In: Bibliotheksforum Bayern 06 (2012), Heft 1, S. 60-62, hier: S. 60.

26 Wie Anm. 1, S. 854.

27 Vgl. Lemanski, Thorsten; Siebert, Irmgard (Hrsg.): Bibliothekare zwischen Verwaltung und Wissenschaft. 200 Jahre Berufsbilddebatte. Frankfurt am Main 2014. 
herkömmlichen als auch die „neuen Tätigkeitsbereiche“ des wissenschaftlichen Bibliotheksdienstes auf, bot aber keine Perspektive, wohin sich der Beruf künftig entwickeln sollte. ${ }^{28}$ Dabei wurde das fachliche Studium als „konstitutiv“ für die anschließende postgraduale Ausbildung bezeichnet. In der anschließenden Diskussion wurde u. a. moniert, dass die IT nicht als eigener, integraler Bereich in das Papier Eingang gefunden hatte, ${ }^{29}$ und vorgeschlagen, ein (noch zu entwerfendes) modernes Berufsbild mindestens an einer objektiven Bestandsaufnahme des realen Arbeitsmarktes zu orientieren. ${ }^{30}$ Die von Konstanze Söllner anhand von Daten aus dem bibliothekarischen Jobportal openbibliojobs durchgeführte Analyse von Stellenausschreibungen für den wissenschaftlichen Bibliotheksdienst (2012-2017) verdeutlicht dabei zweierlei: Erstens ist insgesamt ein quantitativer Anstieg von Stellenausschreibungen für den wissenschaftlichen Dienst zu verzeichnen, während zweitens der Anteil an Fachreferatsstellen rückläufig ist und gleichzeitig der Anteil an Stellen, die im Bereich der „,neuen Tätigkeitsbereiche“ angesiedelt sind, deutlich zunimmt. ${ }^{31}$

Die theoretischen Inhalte der „neuen Tätigkeitsbereiche“ sind durchaus bereits in die Curricula der M.A.-Angebote in Berlin ${ }^{32}$ und Köln ${ }^{33}$ bzw. der Bayeri-

28 Wie Anm. 1, S. 854. Vgl. IG WBS (Hrsg.): Berufsbild Wissenschaftliche Bibliothekarin/Wissenschaftlicher Bibliothekar. 2013. URL: http://www.igwbs.ch/wp-content/uploads/igwbs_berufsbild_dt0901_26052015.pdf [Zugriff: 08. 08. 2018]. Vgl. VDB (Hrsg.): Position des Vereins Deutscher Bibliothekare zur Qualifikation als Wissenschaftliche Bibliothekarin/Wissenschaftlicher Bibliothekar. 2014. URL: https://www.vdb-online.org/wordpress/wp-content/uploads/2014/04/ Position-des-VDB-zur-Qualifikation-als-wissenschaftliche_r-Bibliothekar_in-Final_18.03.2014. pdf [Zugriff: 08. 08. 2018].

29 Vgl. Thesen von Dr. Robert Scheuble. In: Thesen zum Round Table-Gespräch des VDB am 4. März - Teil 2. URL: https://www.vdb-online.org/2015/03/01/thesen-zum-round-tablegesprach-des-vdb-am-4-marz-teil-2/ [Zugriff: 08. 08. 2018].

30 Vgl. Thesen von Felix Lohmeier. In: Thesen zum Round Table-Gespräch des VDB am 4. März Teil 2. URL: https://www.vdb-online.org/2015/03/01/thesen-zum-round-table-gesprach-des-vdbam-4-marz-teil-2/ [Zugriff: 08. 08. 2018]. Vgl. hierzu auch die von Achim Bonte unternommene bedarfsorientierte Differenzierung von zukünftigen Aufgabenbereichen des wissenschaftlichen Dienstes: Bonte, Achim: Befähigung im Wandel. Personalgewinnung und -entwicklung in deutschen Bibliotheken. In: Bibliothek - Forschung und Praxis 41 (2017), Heft 1, S. 115-121, hier: S. $120 \mathrm{f}$.

31 Im Zeitraum vom 17. 07. 2012 bis zum 24. 06. 2015 waren 13,7\% aller auf openbibliojobs ausgeschriebenen Stellen solche des wissenschaftlichen Dienstes. Im Zeitraum vom 25. 06. 2015 bis 31. 07. 2017 betrug dieser Anteil hingegen 16,6\%. Vgl. wie Anm. 1, S. 856 f.

32 Vgl. Petras, Vivien; Gäde, Maria; Rügenhagen, Melanie; Wimmer, Ulla: Die bibliotheks- und informationswissenschaftlichen Masterstudiengänge an der Humboldt-Universität zu Berlin. In: Bibliotheksdienst 51 (2017), Heft 10-11, S. 891-900.

33 Vgl. Oßwald, Achim: Der berufsbegleitende Masterstudiengang Bibliotheks- und Informationswissenschaft (MALIS) der TH Köln. In: Bibliotheksdienst 51 (2017), Heft 10-11, S. 901-912. 
schen Bibliotheksakademie eingegangen. ${ }^{34}$ Unter anderem für die Ausbildung im Bibliotheksreferendariat in München muss jedoch konstatiert werden, dass diese dort nicht grundständig als integraler Teil einer arbeitsmarktorientierten Ausbildung vermittelt, sondern lediglich (mit relativ geringem Zeitanteil) als zusätzlicher Themenbereich unter anderen dem Lehrplan beigefügt werden. Alternativ könnte hier eine grundständige Vermittlung von IT-Kenntnissen mit den informationstechnologischen Dimensionen der „neuen Tätigkeitsbereiche“ wie Forschungsdaten- und Publikationsmanagement, E-Learning, Discovery Systeme oder Metadatenmanagement kombiniert werden. ${ }^{35}$ Die Verantwortlichen an den Ausbildungseinrichtungen sind bei ihren Reformbemühungen selbstverständlich abhängig vom allgemeinen Stand der bibliothekarischen Berufsbilddiskussion, so dass dem Umbau der Curricula die gleiche Hybridität anhaftet wie der gesamten Entwicklung des Bibliothekswesens. Vor diesem Hintergrund ist die aktuelle Überarbeitung der Studienordnung des M.A. „Bibliotheks- und Informationswissenschaft“ am Berliner Institut für Bibliotheks- und Informationswissenschaft zum WS 2018/19 ein begrüßenswerter Schritt in Richtung Profilbildung und stärkerer Gewichtung von IT-Kompetenzen. ${ }^{36}$

34 Vgl. wie Anm. 1, S. $857 \mathrm{f}$.

35 Bspw. könnte sich an eine grundständige Vermittlung geeigneter IT-Kenntnisse der exemplarische Aufbau eines Discovery Systems anschließen, z.B. anhand des Skriptes zur „Bibliotheks- und Archivinformatik“ von Felix Lohmeier. URL: https://felixlohmeier.gitbooks.io/kursbibliotheks-und-archivinformatik/content/ [Zugriff: 08. 08. 2018].

36 Die neue Studienordnung für den nun als M.A. „Information Science“ betitelten Berliner Präsenzstudiengang beinhaltet einen deutlich stärkeren Fokus auf Themen der „neuen Tätigkeitsbereiche“, dabei im Pflichtbereich das Modul „Datenanalyse \& -auswertung“ (mit ca. 8\% des Gesamtdeputats) sowie im Wahlpflichtbereich die Module „Bibliometrie, Informetrie, Szientometrie“, „Information Retrieval“, „Digitale Informationsversorgung“, „Knowledge Discovery in Databases“ und „Digitale Informationsinfrastrukturen“ mit ebenfalls jeweils einem Anteil von ca. $8 \%$ am Gesamtstundenumfang des Studiengangs. Vgl. Fachspezifische Studien- und Prüfungsordnung für den Masterstudiengang Information Science. URL: https://gremien.huberlin.de/de/amb/2018/39/39_2018_ma-information-science_druck.pdf [Zugriff: 08. 08. 2018]. Vgl. hierbei auch die Selbstbeschreibung des Studiengangs: „Das Studium zielt insbesondere auf die Auseinandersetzung mit Themen wie der Optimierung von Informationsprozessen und dem Aufbau, der Gestaltung und der Analyse digitaler Informationsinfrastrukturen und webbasierter Informationssysteme. Es erzeugt Kompetenzen in der Datenanalyse und -verarbeitung, in der Gestaltung und Analyse von Mensch-Maschine-Interaktionen, im Wissens- und Informationsmanagement, in der Informationsvermittlung und wissenschaftlichen Kommunikation in digitalen Informationsumgebungen.“ Ebd. 


\section{Inhaltliche und organisatorische Änderungen in der Ausbildung an der BLB}

Die skizzierten Probleme in der Ausbildung aller drei Qualifikationsniveaus haben in der Regel strukturelle Ursachen, die in der lokalen betrieblichen Ausbildungspraxis nicht aufgelöst werden können - es wurden jedoch im vergangenen Jahr an der BLB an einigen Stellen wichtige inhaltliche Akzentverschiebungen und organisatorische Änderungen vorgenommen. Grundlegend für diese waren die Integration der „neuen Tätigkeitsbereiche“ in die Ausbildung sowie die strikte Orientierung an einer handlungsorientierten und vor allem praxisrelevanten Ausbildung, die sich an der realen Arbeitsmarktsituation der jeweiligen Qualifikationsniveaus orientiert.

\subsection{Informationstechnologie}

Der Bereich der IT ist wohl am schwierigsten in der praktischen Ausbildung zu vermitteln, da die für die Ausbildung einsetzbaren Kapazitäten in den IT-Abteilungen gering sind bzw. weil in den Einrichtungen vor Ort nur das ausgebildet werden kann, was dort auch praktiziert wird. Zudem befinden sich die Curricula der meisten Hochschulen (sowohl im Bereich der B.A.-Studiengänge als auch der M.A.-Programme für Referendar/inn/e/n und Volontär/inn/e/n) momentan in einer Umbauphase, hin zu einer umfangreicheren Vermittlung von IT-Kenntnissen. Die Auszubildenden bringen dabei sehr unterschiedlich entwickelte IT-Erfahrungen mit, so dass an der BLB für alle Qualifikationsniveaus die stufenweise Vermittlung von IT-Kenntnissen einen großen Stellenwert einnimmt. Hierbei spielen auch Inhalte eine Rolle, die prinzipiell ebenso an den theorievermittelnden Ausbildungseinrichtungen vermittelt werden könnten.

An der BLB beginnt die Ausbildung in der IT mit einer umfangreichen und praktischen Einführung in die grundlegenden Aufgaben der hauseigenen IT-Infrastruktur. Dazu gehören die Grundlagen der lokalen Netzwerkarchitektur sowie die Betreuung von Hard- und Software inkl. dem praktischen Kennenlernen und Installieren von verschiedenen Betriebssystemen, z. B. Windows 10, aber auch freie Alternativen wie Ubuntu. Es erfolgt eine Heranführung an die Serververwaltung und -betreuung, die Backup-Prozesse der verschiedenen Server, die konkrete Funktionsweise der WLAN-Infrastruktur, Konzepte der Rechteverwaltung sowie verschiedene von der IT betreute Geräte (Aufsichtsscanner, Bildschirme, Rückgabeautomaten) und Web-Anwendungen (u. a. die technische Betreuung der BLB-Webseite mit Typo3). In diesen Ausbildungsabschnitt fällt auch die Vermitt- 
lung der technischen Grundlagen für die Mitarbeit in den Ausbildungsbereichen der Altbestandsdigitalisierung (im „Team Digitalisierung“) sowie der digitalen Ressourcen (im „Team Netzpublikationen). Das betrifft u.a. die Verwaltung der in der Altbestandsdigitalisierung entstehenden Datenmengen und die administrative Betreuung der Digitalisierungssoftware (hier Visual Library) sowie die Zugriffsverwaltung auf lizenzierte Digitalressourcen (EZ-Proxy, Shibboleth). Je nach persönlicher Interessenslage wird auch kursorisch in Auszeichnungs- und Programmiersprachen (z. B. HTML und PHP) eingeführt. Dieser Ausbildungsabschnitt wird mit kleineren, laufbahnbedingten Abweichungen in allen drei an der BLB ausgebildeten Qualifikationsniveaus in gleichem Maße vermittelt und erfolgt am Beginn der Ausbildung zu einem Zeitpunkt, an dem die elementaren bibliothekarischen Geschäftsgänge schon einmal durchlaufen wurden.

Der zweite IT-relevante Ausbildungsabschnitt beinhaltet systembibliothekarische Tätigkeiten wie die Administration des integrierten Bibliothekssystems, Datenmigrationen sowie Datenimporte und -exporte (u.a. CSV-Dateien, XMLund MARC-Formate sowie zur Bearbeitung geeignete Software und Konzepte wie Catmandu und BaseX). Die Auszubildenden werden eingewiesen sowohl in die Erstellung von Nutzungsstatistiken für z. B. digitale Ressourcen als auch in die technischen Zusammenhänge zwischen Bibliothekssystem, Verbunddatenbank und Discovery System. Vermittelt wird die technische Dimension des Discovery Systems (Katalog plus) und der dahinterstehenden Web-Technologien (VuFind, EDS-Index der Firma Ebsco). Flankierend dazu organisiert eine teamübergreifende Arbeitsgruppe ausbildungsbegleitenden Unterricht zu Discovery Systemen. Um den Praxisbezug zu gewährleisten, erstellen die Auszubildenden selbst konzipierte Datenbankabfragen, die in der systembibliothekarischen Praxis an der BLB auch tatsächlich zum Einsatz kommen; dies können SQL-Abfragen aus dem integrierten Bibliothekssystem für Listen mit den am stärksten in der Ausleihe nachgefragten Titeln sein (sog. „Vormerkrenner“-Listen) oder komplexere Abfragen, die Daten aus dem Lokal- und Verbundsystem kombinieren und über die nach weiteren Verarbeitungsschritten (z.B. mittels Catmandu oder OpenRefine) Aussagen über eingangs angefragte Kriterien gemacht werden können. Positive Erfahrungen hat die BLB auch mit der Übertragung kleinerer systembibliothekarischer Arbeiten an FaMI-Auszubildende machen können. Beispielsweise erarbeitete eine Auszubildende mittels einer selbsterstellten Lokalsystem-Abfrage die Grundlagen für die Darstellung der BLB-Lesesaalsystematik auf der Katalogoberfläche. Dieser IT-Ausbildungsabschnitt steht bei FaMI-Auszubildenden relativ am Ende der Ausbildung, bei den beiden anderen Qualifikationsniveaus schließt sich daran ein weiterer Abschnitt an.

Die LIS-Studierenden im Praxissemester und die Referendar/inn/e/n absolvieren in einem dritten IT-Ausbildungsabschnitt unter systembibliothekarischem 
Mentorat das von Felix Lohmeier dankenswerterweise auf GitHub frei zur Verfügung gestellte Skript zur „Bibliotheks- und Archivinformatik“. ${ }^{37}$ Dabei können sie - die im Skript genutzte Software ist konsequent open access - selbst ein Discovery System aufbauen und lernen dafür nötige Software-Anwendungen kennen (u. a.SRU-Schnittstellen, OpenRefine, Typo3-find, VuFind, Solr und verschiedene Dateiformate). Manche Inhalte wurden dabei auf die spezifische Situation der BLB angepasst. Bereits vorhandene Vorkenntnisse werden zuvor in einem Fragebogen abgeprüft, so dass der mentorierende Systembibliothekar dementsprechend unterschiedliche Akzente setzen bzw. noch einmal im Rahmen konkreter BLB-Anwendungsfälle vertiefen kann. ${ }^{38}$

Die bisherigen Erfahrungen mit der Implementierung der drei IT-Ausbildungsabschnitte sind sehr positiv und haben die Auszubildende/n dazu motiviert, ihre „Schwellenangst“ vor den IT-Themen abzubauen.

\subsection{Netzpublikationen und Altbestandsdigitalisierung}

Neben der Implementierung der drei IT-Ausbildungsabschnitte wurden (v. a. für FaMI-Auszubildende und LIS-Studierende) die Ausbildungsumfänge im Bereich der digitalen Ressourcen und der Altbestandsdigitalisierung stark ausgeweitet.

Im „Team Netzpublikationen“ werden die FaMI-Auszubildenden intensiv mit den spezifischen digitalen Mediengattungen (E-Books, E-Journals, Datenbanken, „graue“ Open Access-Literatur, wie digitale Amtsdruckschriften oder Webseiten), den Zugangsarten (Open Access und die verschiedenen Lizenzierungsmodelle) sowie den Verwaltungs- und Präsentationssystemen (EZB, DBIS und das BadenWürttembergische Onlinearchiv BOA) vertraut gemacht. Die technischen Grund-

37 Vgl. Lohmeier, Felix: Skript zum Kurs „Bibliotheks- und Archivinformatik“ (Herbstsemester 2017) an der HTW Chur. URL: https://felixlohmeier.gitbooks.io/kurs-bibliotheks-und-archivinformatik/content/ [Zugriff: 08. 08. 2018].

38 In einem der umgesetzten BLB-Anwendungsfälle mussten MARC-Titeldaten der BLB aus einem vom Verbund bereitgestellten FTP-Verzeichnis heruntergeladen und dann mit MarcEdit in MARC-XML umgewandelt werden. Anschließend musste VuFind für eine neue Kollektion vorbereitet, die Daten indexiert sowie der Suchindex optimiert werden. Abschließend wurde ein Vergleich bzw. Tests mit den im Discovery System der BLB enthaltenen Daten durchgeführt. In einem anderen Fall mussten die in der Datenbank des Répertoire International des Sources Musicales (RISM) enthaltenen BLB-Daten mittels eines mit PHP selbst zu schreibenden Programms von RISM-XML nach MARC-XML konvertiert werden. Dann wurde mit Catmandu aus der XML-Datei die PICA-Produktionsnummer (PPN) ausgelesen und damit anschließend die in der Verbunddatenbank enthaltenen Daten abgeglichen, welche dann nach bestimmten Kriterien erhalten oder gelöscht wurden. 
lagen dazu werden bereits während des ersten Ausbildungsabschnitts in der IT gelegt (hier Shibboleth und EZ-Proxy). Auch der Umgang mit Nutzungsstatistiken digitaler Ressourcen ist Bestandteil der Ausbildung. Zudem werden die FaMI-Auszubildenden in der Erschließung digitaler Ressourcen ausgebildet. LIS-Studierende im Praxissemester werden an der BLB vorzugsweise im „Team Netzpublikationen“ (sonst in einem anderen Bereich der Abteilung Bestandsaufbau) eingesetzt und dort in den oben genannten Bereichen ausgebildet, mit inhaltlichen Akzentuierungen auf Metadaten- und Lizenzmanagement sowie der Katalogisierung von speziellen digitalen Ressourcen, die insbesondere im Bestandsaufbau einer Landesbibliothek vorkommen. Nach Möglichkeit bearbeiten die LIS-Studierenden dabei auch ein eigenes Projekt: Eine LIS-Studierende war beispielsweise maßgeblich an der Umsetzung eines Workflows für digitale Amtsdruckschriften beteiligt und dabei zuständig für die Erschließung digitaler „grauer Literatur“ in der SWB-Verbunddatenbank, die Prüfung und Verwendung von GND-Daten und die anschließende Bearbeitung in BOA. Sie hat den frisch konzipierten Workflow getestet, angepasst und abschließend dokumentiert.

Der FaMI-Ausbildung im Bereich der Altbestandsdigitalisierung ist ein kurzer Abschnitt im Team „Historische Bestände“ vorgelagert, müssen doch für eine Arbeit in der Digitalisierung wichtiges Wissen zu mittelalterlichen Handschriften, Musikalien, Alten Drucken und anderen Sammlungsgegenständen sowie grundlegende Erschließungskenntnisse auch an FaMIs vermittelt werden. Ein Schwerpunkt in ist die Einführung in die technische Handhabung der Scangeräte, die allgemeinen Digitalisierungsworkflows sowie das Anlegen von Datensätzen in der von der BLB verwendeten Digitalisierungssoftware. Vermittelt werden sowohl die effiziente Präsentation von Altbestandsdigitalisaten (in den Digitalen Sammlungen der BLB, aber auch durch Verlinkungen in der Wikipedia) als auch die Erschließung von Handschriften und Sekundärausgaben in der Verbunddatenbank. LIS-Studierende arbeiten zusätzlich mit bei der grafischen Bearbeitung, Strukturierung und Freischaltung von Altbestandsdigitalisaten, der Erstellung von Anleitungen und einfachen Statistiken sowie der TEI-/XML-Codierung von digitalisierten Briefen.

\subsection{Management und Führung}

Ein häufiges Monitum der praktischen Ausbildung im Referendariat ist die fehlende bzw. zu knappe Ausbildung im Bereich der Direktion. In der BLB nehmen Referendar/inn/e/n nach dem Durchlauf durch die zentralen bibliothekarischen Betriebsabteilungen (in etwa nach einem Drittel der praktischen Ausbildungszeit) bis zum Ende der Ausbildung an der wöchentlich tagenden Lenkungsrunde 
der Direktion teil und werden damit umfangreich an typische Leitungs- und Führungsaufgaben des wissenschaftlichen Bibliotheksdienstes herangeführt. Sie werden an ausgewählten, die Direktion berührenden Sitzungsterminen beteiligt, absolvieren einen längeren Aufenthalt in der Verwaltung und arbeiten in verschiedenen Stabstellen mit wie dem Controlling, der Presse- und Öffentlichkeitsarbeit oder dem Baureferat. In Gesprächen mit der Direktion werden die dort gemachten Erfahrungen reflektiert.

LIS-Studierende im Praxissemester werden hingegen stark in die Tagesaufgaben des Bereichs der Abteilung Bestandsaufbau eingebunden, in dem sie prioritär eingesetzt sind. Dabei lernen sie die konkreten Verwaltungs- und Führungsaufgaben der Teamleitung praktisch kennen, nehmen an den Sitzungen des Teams sowie der Teamleiter/innen teil, sind am Austausch mit der Abteilungsleitung beteiligt und reflektieren diese Erfahrungen und Lernprozesse in gemeinsamen Gesprächen mit dem/der Teamleiter/in. Auch leisten die LIS-Studierenden nach Möglichkeit konzeptionelle Zuarbeiten für die Teamleitungen.

\subsection{Weitere inhaltliche Änderungen in der Ausbildung an der BLB (nach Qualifikationsniveaus)}

Die vergleichsweise lange, in der Regel dreijährige Ausbildungsdauer der FaMIAuszubildenden erleichtert die Integration der „neuen Tätigkeitsbereiche“ in die betrieblichen Ausbildungspläne. Zwar bedarf die aus dem Jahr 1998 stammende Ausbildungsordnung einer grundlegenden Anpassung an die bibliothekarischen Entwicklungen der letzten 20 Jahre, ist aber in weiten Teilen so flexibel und offen gehalten, dass die „neuen Tätigkeitsbereiche“ integrierbar sind. ${ }^{39}$ Jene Zukunftsthemen, die in der betrieblichen Praxis der BLB kaum vorkommen (wie z. B. der universitär geprägte Open Access-Bereich oder Forschungsdatenmanagement), müssen aufgrund ihrer großen Bedeutung für das Bibliothekswesen extern vermittelt werden. Die FaMI-Auszubildenden der BLB lernen diese Bereiche künftig im Rahmen einer informellen Ausbildungskooperation an der KIT-Bibliothek kennen. Solche informellen, lokalen Kooperationen wären sicherlich auch in den kommunikativ orientierten „neuen Tätigkeitsbereichen“ für FaMIs wünschenswert - zu denken wäre hierbei an Kooperationen mit öffentlichen Bibliotheken für den Bereich des bibliothekarischen Veranstaltungsmanagements oder erfolgreicher Umsetzung von Social Media-Konzepten.

39 Vgl. wie Anm. 5, S. 944. 
Um der unzureichenden Vermittlung von Katalogisierungs- und Regelwerkskunde an der Berufsschule entgegenzuwirken, führt die BLB erstmals (wieder) im Sommer 2019 einen zweiwöchigen ausbildungsbegleitenden RDA-Unterricht durch, der auch den FaMI-Auszubildenden anderer Einrichtungen offensteht. Daneben sind andere Bereiche, wie die Funktionsweise von Discovery Systemen oder umfassende Recherchekompetenzen, nur schwerlich von den Berufsschulen $\mathrm{zu}$ vermitteln und werden deshalb in der BLB ebenfalls durch gesonderten ausbildungsbegleitenden Unterricht in die Ausbildung integriert.

Dem Problem der zu geringen Zahl an Bewerbungen für FaMI-Ausbildungsstellen kann lokal nur sehr bedingt abgeholfen werden, weshalb die Forderung der ekz nach einer koordinierten Aufklärung der berufsberatenden Stellen (wie u. a. Arbeitsagenturen) über den aktuellen Wandel des Bibliotheksberufes ein begrüßenswerter Schritt wäre, den einstellenden Einrichtungen geeignetere Bewerber/innen zukommen zu lassen. ${ }^{40}$ Zudem ist es m.E. sinnvoll, den Fokus bei der Suche nach geeigneten Bewerber/inne/n auf Personen mit mittleren Bildungsabschlüssen zu legen, auf die der Komplexitätsgrad einer beruflichen Ausbildung eigentlich zugeschnitten ist. Dazu ist eine engere Zusammenarbeit mit den (Real-)Schulen im Rahmen der berufsorientierenden Praktika nötig, ${ }^{41}$ um bereits an dieser Stelle geeignete Praktikant/inn/en zu gewinnen. ${ }^{42}$ Solche Praktika werden jedoch nicht an allen wissenschaftlichen Bibliotheken in wünschenswertem Umfang angeboten. ${ }^{43}$ Der Auftritt auf lokalen Berufs- und Ausbildungsmessen, in den lokalen Medien sowie Social Media-Kanälen und überregionalen Jobportalen, der idealerweise zwischen den örtlichen bibliothekarischen Einrichtungen koordiniert sein sollte, ist zeit- und kostenintensiv, direkte Auswirkungen sind hingegen nur schwer messbar - nichtsdestotrotz muss mangels Alternativen umfangreich in diese Art von Recruitment investiert werden. ${ }^{44}$ Wie eine moderne Recruitment-Kampagne für den öffentlichen Dienst aussehen

40 Vgl. wie Anm. 10.

41 Vgl. wie Anm. 11, S. 472.

42 In Baden-Württemberg finden diese berufsorientierenden Praktika für Gymnasiast/inn/en im Rahmen der Berufs- und Studienorientierung am Gymnasium (BOGY) in der 9. bzw. 10. Jahrgangsstufe, für Realschüler/innen im Rahmen der Berufsorientierung für Realschulen (BORS) in der Regel in der 9. Jahrgangsstufe statt. Vgl. auch die Forderung im Rahmen der Umfrage des dbv-Landesverbandes Baden-Württemberg, „mehr Praktika“ anzubieten. Vgl. wie Anm. 4, S. 14. 4311 von 25 befragten wissenschaftlichen Bibliotheken in Baden-Württemberg bieten keine berufsorientierenden Praktika für Realschüler/innen, 7 von 25 keine für Gymnasiast/inn/en an. Vgl. wie Anm. 4, S. 13.

44 Vgl. beispielsweise die Ergebnisse des Hands-On Labs zu neuen Perspektiven im Personalmarketing auf dem Bibliothekartag 2018: Erdmenger, Tanja; Krass, Ulrike; Langenkamp, Karin; Winalke, Jens: Neue Perspektiven im Personalmarketing. Wie Bibliotheken ihre Mitarbeiten- 
kann, hat beispielsweise vor kurzem die bayerische Polizei mit ihrer CrossmediaKampagne „Mit Sicherheit anders“ eindrucksvoll illustriert. ${ }^{45}$

Weit übersichtlicher stellt sich die Situation bei den LIS-Studierenden dar: Im Verlauf eines typischen B.A.-Studiums bieten sich für die Bibliotheken in der Regel zwei Möglichkeiten, die späteren Bewerber/innen kennenzulernen und deren Hochschulausbildung mit Praxiserfahrungen anzureichern: Zum einen das (relativ kurze) Orientierungspraktikum zu Beginn des Studiums und zum anderen das Praxissemester im fünften Semester. Dabei haben die Bibliotheken nur sehr begrenzten Einfluss auf die personelle Auswahl, es sei denn, es liegen mehr Praktikumsbewerbungen vor als Ausbildungskapazitäten zur Verfügung stehen. Neben Bestandsaufbau mit Schwerpunkt digitale Ressourcen, Altbestandsdigitalisierung und IT ist nach Interessenslage und Möglichkeiten auch der Schulungsbereich eine weitere Einsatzmöglichkeit für LIS-Studierende im Praxissemester an der BLB.

Die Referendar/inn/e/n an der BLB werden neben dem Kennenlernen der Bibliotheksbereiche, der Vermittlung von IT-Kenntnissen und der vertieften Praxiserfahrung im Bereich Führung und Management schwerpunktmäßig auf Feldern ausgebildet, die in der innerbibliothekarischen Diskussion der vergangenen Jahre zur Disposition gestellt wurden, jedoch m. E. konstitutiv für den wissenschaftlichen Dienst sind: die Erwerbung von Medien sowie deren inhaltliche Erschließung, dies üblicherweise im Rahmen der eigenverantwortlichen Übernahme des Fachreferats im studierten Fach sowie eines fachfremden Fachreferats. Dazu gehört neben der wertenden Auswahl von zu sammelnden Medien aller Gattungen und physischen Formen ${ }^{46}$ sowie der Sacherschließung nach RSWK (unter Zuhilfenahme von halbautomatischen Web-Anwendungen wie dem Digitalen Assistenten) auch die Durchführung von Informationskompetenz-Schulungen aller Art. Da

den finden. Konferenzveröffentlichung 107. Deutscher Bibliothekartag in Berlin 2018. URN: urn:nbn:de:0290-opus4-157599 [Zugriff: 08. 08. 2018]. Vgl. wie Anm. 12, S. 481.

45 Wobei hier natürlich ungleich umfassendere personelle und finanzielle Ressourcen zur Verfügung stehen als im Bibliothekswesen. Vgl. die Kampagnen-Webseite der bayerischen Polizei „Mit Sicherheit anders“. URL: https://www.mit-sicherheit-anders.de/ [Zugriff: 08. 08. 2018]. Vgl. wie Anm. 11, S. 471. Hier wurde die Personalgewinnungskampagne der Bundeswehr („Mach, was wirklich zählt“) sowie die Imagekampagne des Deutschen Handwerkskammertags (DHKT) näher beleuchtet.

46 Vgl. hierzu u.a. Kempf, Klaus: Sammlung ade? In: Ceynowa, Klaus (Hrsg.) Bibliotheken: Innovation aus Tradition. Rolf Griebel zum 65. Geburtstag, München 2014, S. 371-408. Vgl. Ceynowa, Klaus: Was zählt und was stört - Zukunftsperspektiven der Bibliothek. Zwischenrufe eines Erfahrungsübersättigten. In: Bonte, Achim; Rehnolt, Juliane (Hrsg.): Kooperative Informationsinfrastrukturen als Chance und Herausforderung. Thomas Bürger zum 65. Geburtstag. Berlin 2018, S. 53-69, insbesondere S. 62-67. 
mit den oben skizzierten Änderungen die Ausbildungsinhalte stark angewachsen sind, mussten im Gegenzug der zeitliche Umfang sowohl in den bibliothekarischen Kernbereichen als auch bei den externen Praktika reduziert werden.

\subsection{Organisatorische Änderungen in der Ausbildung an der BLB}

Organisatorische Grundlage für die inhaltlichen Änderungen in der bibliothekarischen Ausbildung an der BLB war die Formierung einer „Ausbildungsrunde“, die sich aus den ausbildenden Fachkräften der bibliothekarischen Kernbereiche (inkl. der Bereiche, in denen die „neuen Tätigkeiten“ schwerpunktmäßig anfallen), der allgemeinen und systembibliothekarischen IT sowie der Ausbildungsleitung zusammensetzt und einmal im Monat grundlegende Fragen der Ausbildung diskutiert. Neben der Implementierung der die „neuen Tätigkeiten“ ausbildenden Bereiche in die Ausbildung hat sich die Runde umfassend mit dem aus der FaMI-Ausbildungsordnung abgeleiteten baden-württembergischen Ausbildungsrahmenplan beschäftigt. In einem mehrstufigen Arbeits- und Diskussionsprozess hat sie für jeden Ausbildungsbereich von den Richt- und Groblernzielen abgeleitete, betriebsspezifische Feinlernziele formuliert und in einem gemeinsamen „Feinlernziel-Plan“ für die FaMI-Ausbildung an der BLB zusammengetragen. Auf diesem Weg konnten bestehende Lücken gefüllt, neue Ausbildungsbereiche inhaltlich erschlossen sowie Synergieeffekte sinnvoll gesteuert werden. Ein strukturiertes Feedback-System trägt dabei seinen Teil zur Qualitätssicherung der Ausbildung aller Qualifikationsniveaus bei, wobei an der BLB nicht nur die Ausbilder/innen die Auszubildenden bewerten, sondern ebenso umgekehrt die Auszubildenden die Möglichkeit haben, die Ausbildung des jeweils erfolgten Ausbildungsabschnitts zu evaluieren sowie eine jedem Feedback-Gespräch mit der Ausbildungsleitung vorhergehende Selbstevaluierung vornehmen. Zudem organisiert die BLB ein Vernetzungstreffen der Ausbildungsverantwortlichen der (öffentlichen und wissenschaftlichen) Bibliotheken in und um Karlsruhe. Hier werden Erfahrungen (vor allem im Bereich der FaMI-Ausbildung) strukturiert ausgetauscht, lokale Aktivitäten rund um die Ausbildung koordiniert und Möglichkeiten zum Austausch von Lehr- und Ausbildungsmaterialien geschaffen. Seit dem Ausbildungsjahr 2017/18 bietet die BLB zudem als eine der wenigen wissenschaftlichen Bibliotheken in Baden-Württemberg ein FSJ Kultur an. ${ }^{47}$ Die

47 Nur 2 von 25 befragten wissenschaftlichen Bibliotheken in Baden-Württemberg haben 2017 ein FSJ Kultur angeboten, vgl. wie Anm. 4, S. 13. 
FSJ Kultur-Freiwilligen werden primär in den Bereichen Presse- und Öffentlichkeitsarbeit sowie im Schulungsbereich eingesetzt, wobei sich in vielerlei Hinsicht Austausch- und Kooperationsmöglichkeiten mit den bibliothekarischen Auszubildenden ergeben und wichtige Synergieeffekte entstehen. Dies gilt ebenso für die Auszubildenden des ebenfalls an der BLB ausgebildeten Berufs des Fachinformatikers (Systemintegration).

\section{Ausblick}

Die hier skizzierten Erfahrungen bei der Integration der „neuen Tätigkeiten“ in die Ausbildung der BLB werden sowohl von der Ausbildungsleitung als auch von den Auszubildenden selbst als insgesamt sehr positiv beurteilt, insbesondere hinsichtlich des auf diesem Wege erfolgenden verstärkten Erwerbs von arbeitsmarktrelevanten Qualifikationen. Zukünftig wird in der Ausbildung an der BLB ein noch stärkerer Fokus auf die Vernetzung mit anderen ausbildenden Bibliotheken gelegt werden, um noch besser von den dadurch entstehenden Synergieeffekten beim Austausch von inhaltlichen Konzepten, organisatorischen Erfahrungen und Ausbildungsmaterialien profitieren zu können.

Auch weiterhin wird - auf allen Qualifikationsniveaus - die Gewinnung von geeigneten Auszubildenden die größte Herausforderung für die bibliothekarische Ausbildung darstellen. Insbesondere vor diesem Hintergrund muss die Kooperation der ausbildenden Bibliotheken mit den theorievermittelnden Ausbildungseinrichtungen weiter intensiviert werden bzw. müssen diese ihre Inhalte noch praxisorientierter gestalten. Sehr zu begrüßen sind in diesem Zusammenhang die ersten vorgeschlagenen Maßnahmen der verbändeübergreifenden AG Personalgewinnung zur Vermittlung eines modernen Images von Bibliotheken und eines modernen Berufsbildes sowie die Erstellung von Materialien zur Personalgewinnung vor Ort. ${ }^{48}$

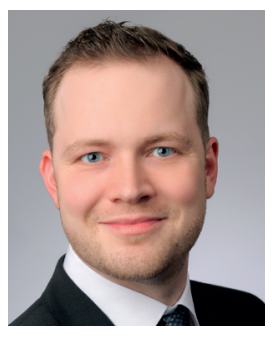

\section{Dr. Michael Fischer}

Badische Landesbibliothek

Erbprinzenstr. 15

76133 Karlsruhe

Deutschland

Mail: fischer@blb-karlsruhe.de

48 Vgl. wie Anm. 11, S. 471 f. 